\title{
A Standard Generator/Parity Check Matrix for Codes from the Cayley Tables Due to the Non-associative (123)-Avoiding Patterns of AUNU Numbers
}

\author{
Ibrahim A.A ${ }^{1}$, Chun P.B ${ }^{2, *}$, Abubakar S.I ${ }^{3}$, Garba A.I ${ }^{1}$, Mustafa. $A^{1}$ \\ ${ }^{1}$ Department of Mathematics, Usmanu Danfodiyo University Sokoto, Nigeria \\ ${ }^{2}$ Department of Mathematics, Plateau State University Bokkos, Nigeria \\ ${ }^{3}$ Department of Mathematics, Sokoto State University Sokoto, Nigeria
}

Copyright $\bigcirc 2016$ by authors, all rights reserved. Authors agree that this article remains permanently open access under the terms of the Creative Commons Attribution License 4.0 international License.

\begin{abstract}
In this paper, we aim at utilizing the Cayley tables demonstrated by the Authors[1] in the construction of a Generator/Parity check Matrix in standard form for a Code say $\mathbf{C}$ Our goal is achieved first by converting the Cayley tables in [1] using Mod2 arithmetic into a Matrix with entries from the binary field. Echelon Row operations are then performed (carried out) on the matrix in line with existing algorithms and propositions to obtain a matrix say $\mathbf{G}$ whose rows spans $\mathbf{C}$ and a matrix say $\mathbf{H}$ whose rows spans $\mathbf{C}^{\perp}$, the dual code of $\mathbf{C}$, where $\mathbf{G}$ and $\mathbf{H}$ are given as, $G=\left(I_{k} \mid X\right)$ and $\mathrm{H}=\left(-\mathrm{X}^{\mathrm{T}} \mathrm{I}_{\mathrm{n}-\mathrm{k}}\right)$. The report by Williem (2011) that the adjacency Matrix of a graph can be interpreted as the generator matrix of a Code [3] is in this context extended to the Cayley table which generates matrices from the permutations of points of the AUNU numbers of prime cardinality.
\end{abstract}

Keywords Carley Tables, AUNU Permutation Patterns, Generator Matrix, Parity Check Matrix, Standard Form, Pattern Avoidance, Echelon Row Operation, Non-associative, Non-commutative

\section{Introduction}

In Coding theory, the generator matrix of a Code plays an important role. Once the generator matrix of a code is known, such a code can easily be encoded and decoded, since procedures for obtaining the parity check matrix say of the code from the generator matrix is obvious through existing algorithms and theorems. As such, we shall not be out of place to mention here that once we have the generator matrix for a particular code, then a message can be encoded, decoded and analyzed by the matrix.

The generation and analysis of some small classes of linear and cyclic codes from the adjacency matrices of
Eulerian graphs due to AUNU patterns had been reported in [2] and [3]. This special class of the (132) and (123)-avoiding class of permutation patterns which were first reported [4], where some group and graph theoretic properties were identified, had enjoyed a wide range of applications in various areas of applied Mathematics. In[1], we described how the non-associative and non-commutative properties of the patterns can be established using their Cayley tables where a binary operation was defined to act on the (132) and (123)-avoiding patterns of the AUNU numbers using a pairing scheme.

In this paper, we utilize the Cayley tables generated, first as matrices, then as matrices over the binary field and lastly transform such matrices into generator/parity check matrices for some codes in standard form $G=\left(I_{k} \mid X_{n-k \times k}\right) / H=\left(-X^{T} \mid I_{n-}\right)$.

We review some basic concepts and propositions for the easy understanding of this paper.

\subsection{Permutation Patterns:}

An arrangement of the objects $1,2, \ldots, n$ is a sequence consisting of these objects arranged in any order. When in addition, a particular order is desired, such an arrangement becomes an ordered permutation governed by a pattern $\sigma$ and such a permutation pattern $\sigma \in S_{n}$ naturally results into a certain arrangement of $1,2, \ldots, n$ given by

$$
\sigma(1) \sigma(2) \cdot . \sigma(n)
$$

which is called the arrangement associated with a permutation pattern $\sigma$ of points of a non-empty set,

$$
\Omega=\{1,2, \ldots n\}
$$

Given a sequence $\pi$ consisting of $n$ elements arranged in a given pattern and another sequence $\sigma$ having $m$ elements such that $m<n$, then $\sigma$ is said to be 
contained as a pattern in $\pi$ provided $\pi$ has a subsequence which its order is isomorphic to $\sigma$. If $\pi$ does not contain $\sigma$, it is said to avoid it. The set of all $\sigma$-avoiding permutation is denoted by $S_{n}(\sigma)$.

It is useful to differentiate between a subsequence and a subword. For instance, if $\sigma=4132 \in S_{4}, \pi=78364521 \in S_{8}$ contains $\sigma$ as a subword since $\rho(8364)=4132$. However, $\pi=54321 \in S_{5}$ does not contain $\sigma$ as a subword although it does contain it as a subsequence. Occurrences of subwords can be overlapped. As an example, the sequence $5716243 \in S_{7}$ contains two occurrences of $\sigma=7162$ and 6234. Determination of $\left|S_{n}(\sigma)\right|$ has remained a hard and intractable problem for a given $\sigma$ containing more than three elements. This is among the reasons that motivate the Author to construct some class of pattern-avoiding permutations using some special subword (instead of subsequences) governed some succession scheme (Ibrahim, 2005). Thus $A_{n}(132)$ in this context, represents the subwords of length $n \geq 3$ that are (132) -avoiding in $A_{n}$ being the set of strictly consecutive succession scheme containing pairs $i, j$ such that

$$
j=i+1 \quad i, j \in \Omega \text {. }
$$

\section{Methodology}

We consider the Cayley table below, which is constructed using $A_{n}(132)$ for $n=5$ as in [2]

Table 1. Cayley Table for $n=5$ showing generated points of $\Omega$ as permutations of (132) and (123)-avoiding patterns of AUNU scheme under the action of $\Theta$.

\begin{tabular}{|l|l|l|l|l|l|}
\hline$\Theta$ & 1 & 2 & 3 & 4 & 5 \\
\hline 1 & 1 & 3 & 5 & 2 & 4 \\
\hline 2 & 1 & 4 & 2 & 5 & 3 \\
\hline 3 & 1 & 5 & 4 & 3 & 2 \\
\hline
\end{tabular}

We now convert the entries of the Cayley table above to the binary system using Modulus 2 arithmetic. The table thus becomes;

\begin{tabular}{|c|l|l|l|l|l|}
\hline$\Theta$ & 1 & 2 & 3 & 4 & 5 \\
\hline 1 & 1 & 1 & 1 & 0 & 0 \\
\hline 2 & 1 & 0 & 0 & 1 & 1 \\
\hline 3 & 1 & 1 & 0 & 1 & 0 \\
\hline
\end{tabular}

The above Cayley table is the matrix $A$ below;

$$
A=\left[\begin{array}{lllll}
1 & 1 & 1 & 0 & 0 \\
1 & 0 & 0 & 1 & 1 \\
1 & 1 & 0 & 1 & 0
\end{array}\right]
$$

\section{Proposition:}

The matrix $A=\left[\begin{array}{lllll}1 & 1 & 1 & 0 & 0 \\ 1 & 0 & 0 & 1 & 1 \\ 1 & 1 & 0 & 1 & 0\end{array}\right]$ from the Cayley table 1 above is equivalent to a matrix $G^{I}=\left[I_{k} \mid X_{k \times n-k}\right]$, the generator matrix in standard form of a code $C$ spanned by the rows of A.

Proof: suppose $A$ above is a generator matrix for some linear code $C$. Then $C$ has dimension $k=3$ and length $n=5$. According to an established result, the rows of a generator matrix $G$ are independent, which is obvious for our $G=A$ above. Now, since every generator matrix can either be put in standard form or is equivalent to a generator matrix in standard form i.e $\left[I_{k} \mid X_{k \times n-k}\right]$. Apply the following Reduced Row Echelon form (RREF) operations on A; $\quad$ i. $R_{2}=R_{3}$ and ii. $R_{3}=R_{2}+R_{3}$ clearly gives ${ }_{u s} G^{*}=\left[X_{k \times n-k} \mid I_{k}\right]=X_{3 \times 5-3}\left|I_{3}=X_{3 \times 2}\right| I_{3}$ where $X_{k \times n-k}=X_{3 \times 2}\left[\begin{array}{ll}1 & 1 \\ 1 & 1 \\ 0 & 1\end{array}\right]$ and $I_{k}=I_{3}$ the identity matrix of order 3. On juxtaposition of $G^{*}$, we obtain $G^{I}=\left[I_{k} \mid X_{k \times n-k}\right]$ which is our required result.

Next, we transform the $A$ to a matrix $G$ in standard form using the above proposition. Now, applying the following row operations;

$$
\begin{aligned}
& \text { i. } R_{2}=R_{3} \text { on } A \rightarrow A=A^{I}=\left[\begin{array}{lllll}
1 & 1 & 1 & 0 & 0 \\
1 & 1 & 0 & 1 & 0 \\
1 & 0 & 0 & 1 & 1
\end{array}\right] \text { and } \\
& \text { ii. } R_{3}=R_{2}+R_{3} \text { on } A^{I} \rightarrow A^{I}=A^{I I}=\left[\begin{array}{lllll}
1 & 1 & 1 & 0 & 0 \\
1 & 1 & 0 & 1 & 0 \\
0 & 1 & 0 & 0 & 1
\end{array}\right]
\end{aligned}
$$

Clearly, $A^{I I}$ can be written as $A^{I I}=G^{I}=\left[\begin{array}{ll|lll}1 & 1 & 1 & 0 & 0 \\ 1 & 1 & 0 & 1 & 0 \\ 0 & 1 & 0 & 0 & 1\end{array}\right]=\left[X_{k \times n-k} \mid I_{k}\right]$ and on 
juxtaposition of $G^{I}$, we have

$$
G=\left[\begin{array}{lll|ll}
1 & 0 & 0 & 1 & 1 \\
0 & 1 & 0 & 1 & 1 \\
0 & 0 & 1 & 0 & 1
\end{array}\right]=\left[I_{k} \mid X_{k \times n-k}\right]=\left[\begin{array}{lllll}
1 & 0 & 0 & 1 & 1 \\
0 & 1 & 0 & 1 & 1 \\
0 & 0 & 1 & 0 & 1
\end{array}\right]
$$

which is the required generator matrix in standard form for a Code of length $n=5$ and dimension

$$
k=3 \quad, \quad \text { Efficiency }=\text { Rate }=\frac{3}{5} \quad \text { and }
$$

and 3 message digits with 2 parity check digits.

For the dual code $C^{\perp}$ of the code $C$ whose generator matrix in standard form is $H$ (also the parity check matrix of $C$ ). We define a matrix $H^{I}=\left[-X^{T} \mid I_{n-k}\right]$ from $G^{I} \quad$ above. i.e $H^{I}=\left[\begin{array}{lll|ll}1 & 1 & 0 & 1 & 0 \\ 1 & 1 & 1 & 0 & 1\end{array}\right]$ and on juxtaposing $H^{I}$, we have $H=\left[\begin{array}{ll|lll}1 & 0 & 1 & 1 & 0 \\ 0 & 1 & 1 & 1 & 1\end{array}\right]$. And we conclude that $H$ is the parity check matrix of $\mathrm{C}$ and the generator matrix of the orthogonal complement of $\mathrm{C},\left(C^{\perp}\right)$.

\section{Findings}

The Cayley table 1, when interpreted as a binary Matrix using Modulus 2 arithmetic, has been transformed into a generator matrix in standard form for a code of length $n=5$, dimension $k=3 \quad, \quad$ Efficiency $=$ Rate $=\frac{3}{5}$. Moreover, using existing algorithms and results, the parity check matrix $\mathrm{H}$ of the code which is also the generator matrix of the dual code $C^{\perp}$ has also been obtained.

\section{Conclusions}

The Cayley tables due to the non-associative property of the (123)- avoiding class of AUNU permutation pattern has found suitable application in determining the generator /parity check matrices in standard form for some classes of codes.

\section{Acknowledgements}

We acknowledge the support enjoyed in this research endeavour by the Institution Based Research (IBR) TETFund Grand 2015, of the Sokoto State University, Sokoto, Nigeria.

\section{REFERENCES}

[1] Ibrahim A.A., \& Abubakar S.I. (2016) Non-Associative Property of 123-Avoiding Class of Aunu Permutation patterns. Advances in Pure Mathematics, 6, 51-57. http://dx.doi.org/10.4236/apm.2016.62006.

[2] Chun P.B, Ibrahim A.A, \&Garba A.I, (2016) Algebraic theoretic properties of the avoiding class of AUNU permutation patterns: Application in the generation and analysis of linear codes. International Organization for Scientific Research (IOSR), Journal of Mathematics 12 (1) pp $1-3$.

[3] Chun P.B, Ibrahim A.A, \&Garba A.I, (Accepted; yet to be published) Algebraic Theoretic Properties of The Non-Associative Class of (132)-Avoiding Patterns of Aunu Permutations: Applications In The Generation And Analysis Of A General Cyclic Code. HORIZON RESEARCH Publishing corporation, USA.

[4] Ibrahim A.A \&Audu M.S. (2005) some group theoretic properties of certain class of (123) and (132) - avoiding pattern of certain numbers; An enumeration scheme, African journal of natural Sciences 819-84.

[5] Hoffman, D.G., Leonard, D.A., Lindner, C.C., Phelps, K.T., Rodger, C.A., \& Wall, J.R (1992), Coding Theory: The Essentials. Mercel Dekker, Inc, 1992.

[6] Willem, H. H (2011) Matrices for graphs, Designs and Codes, (NATO Science for Peace and security, series 29 Information Security), Coding theory and Related Combinatorics, IOS press, 2011.

[7] Ibrahim M, Ibrahim A.A, Yakubu, M.A. (2012) Algebraic theoretic properties of the (132)-avoiding class of Aunu patterns application in Eulerian graphs, Journal of Science and Technology Research, 2012.

[8] Vasantha W.B, Florentin .S., \& Ilanthenral .K. (2010) Supper Special Codes Using Supper Matrices. Infolearnquest, Ann Arbor, 2010.

[9] Usman A. \& Ibrahim A.A (2011) A new generating function for AUNU patterns: Application in integer group Modulo $n$.Nigerian Journal of Basic and Applied Sciences 19 (1)

[10] Bruck R.H (1958) A survey of the binary system. Springer Berlin 